\title{
Cosmic Birefringence Triggered by Dark Matter Domination
}

\author{
Shota Nakagawa $\odot,{ }^{1}$ Fuminobu Takahashi, ${ }^{1,2}$ and Masaki Yamada ${ }^{1,3}$ \\ ${ }^{1}$ Department of Physics, Tohoku University, Sendai, Miyagi 980-8578, Japan \\ ${ }^{2}$ Kavli IPMU (WPI), UTIAS, The University of Tokyo, Kashiwa, Chiba 277-8583, Japan \\ ${ }^{3}$ Frontier Research Institute for Interdisciplinary Sciences, Tohoku University, Sendai, Miyagi 980-8578, Japan
}

(Received 24 March 2021; revised 19 July 2021; accepted 8 October 2021; published 28 October 2021)

Cosmic birefringence is predicted if an axionlike particle (ALP) moves after the recombination. We show that this naturally happens if the ALP is coupled to the dark matter density because it then acquires a large effective mass after the matter-radiation equality. Our scenario applies to a broad range of the ALP mass $m_{\phi} \lesssim 10^{-28} \mathrm{eV}$, even smaller than the present Hubble constant. We give a simple model to realize this scenario, where dark matter is made of hidden monopoles, which give the ALP such a large effective mass through the Witten effect. The mechanism works if the ALP decay constant is of order of the grand unified theory scale without a fine-tuning of the initial misalignment angle. For smaller decay constant, the hidden monopole can be a fraction of dark matter. We also study the implications for the QCD axion, and show that the domain wall problem can be solved by the effective mass.

DOI: 10.1103/PhysRevLett.127.181103

Introduction.-Axions are ubiquitous in string theory and are known to have interesting effects on various observables, despite the fact that their interactions are suppressed by large mass scales such as the string scale. For instance, the collective excitations, like coherent oscillations, can be induced during the evolution of the Universe [1-3], which may explain all or part of dark matter (DM). A large number of axions are also produced by the decay of moduli fields, contributing to the effective number of neutrino species [4-9]. Very light axions can extract angular momentum from rotating black holes via superradiance [10-15]. In addition to gravitational interactions, axions can have interactions with the standard model particles. Among them, an anomalous coupling with photons is known to cause very diverse phenomena in cosmology and astrophysics, such as $\mathrm{x}$-ray or $\gamma$-ray emission from the decay of axions [16-20] (see Refs. [2123] for early works on a similar subject) and cosmic birefringence $(\mathrm{CB})$, i.e., the rotation of the photon polarization plane [24-31]. Axions coupled to photons are often referred to as axionlike particles (ALPs).

Recently, it was reported in Ref. [32] that the Planck 2018 polarization data of cosmic microwave background (CMB) favor a nonzero value of isotropic $\mathrm{CB}$, with statistical significance of $2.4 \sigma$, based on the novel method [33-35]. The suggested rotation angle of the CMB polarization is (a

Published by the American Physical Society under the terms of the Creative Commons Attribution 4.0 International license. Further distribution of this work must maintain attribution to the author(s) and the published article's title, journal citation, and DOI. Funded by SCOAP ${ }^{3}$. positive $\beta$ corresponds to clockwise rotation for an observer.) $\beta=0.35 \pm 0.14 \mathrm{deg}$, and future observations will reduce statistical uncertainties by more than 1 order of magnitude [36]. One plausible explanation of the isotropic $\mathrm{CB}$ is an ALP, which starts to move during or after the recombination epoch. (Another possibility is the axion domain wall, which works for heavier ALP masses, and predicts peculiar anisotropic CB [37].) The ALP mass relevant for this scenario is in the range of $\left(10^{-33} \mathrm{eV}, 10^{-28} \mathrm{eV}\right)$, because, for heavier or lighter ALP masses, a large enhancement of the ALP-photon coupling is required [38].

Interestingly, the isotropic $\mathrm{CB}$ implied by the current observation is very natural from a theoretical point of view. It is determined by the ratio of the change in the ALP to its decay constant, and is not sensitive to the decay constant itself. The observed rotation angle suggests that the ALP has changed by about the decay constant from the time of recombination to the present. In other words, no fine-tuning of the initial value is necessary. Even so, one might wonder why the ALP has started moving at such a special timing in the history of the Universe. The timing of when the ALP begins to oscillate is determined by the balance between the Hubble parameter and the ALP mass. This may seem ad hoc, if there were not for any particular theoretical reason. One might think that, if the ALP masses are logarithmically distributed on each scale as suggested in the string axiverse [10], this is naturally explained (see Ref. [39] for a recent analysis). However, it is by no means obvious that this is indeed the case, since it is not known how many axions actually exist in the low energy and whether they are coupled to photons in the standard model. In fact, if the string axion masses are logarithmically distributed, the smallness of the mass itself may not be a 
problem, but it actually implies that there is no natural lower bound on the axion mass. Therefore, it can still be regarded as a "coincidence," i.e., some kind of fine-tuning problem regarding the timing of the ALP oscillations.

In this Letter, we present a scenario that explains the reason for the coincidence of the recent ALP oscillations. Our scenario is based on the observational fact that the recombination and matter-radiation equality occur in close proximity. We point out that if the ALP starts to move via coupling with the DM density after the matter-radiation equality, it naturally induces the isotropic $\mathrm{CB}$ for a broader range of the mass. From the point of view of a low-energy effective field theory, such a phenomenon occurs if the ALP acquires an effective mass of the order of the Hubble parameter during the matter-dominated era. Such an effective mass may come from the interaction between the ALP and (dark) matter with gravitational strength. We will present a concrete model in which the ALP acquires an effective mass via the Witten effect of hidden monopole DM. We discuss that, if the QCD axion acquires a similar effective mass, the isocurvature and domain wall problems can also be solved.

Cosmic birefringence from ALP dynamics.-We consider an (almost) massless ALP that couples to photons via anomaly:

$$
\mathcal{L} \supset-c_{\gamma} \frac{\alpha}{4 \pi} \frac{\phi}{f_{\phi}} F_{\mu \nu} \tilde{F}^{\mu \nu} \equiv-\frac{1}{4} g_{\phi \gamma \gamma} \phi F_{\mu \nu} \tilde{F}^{\mu \nu},
$$

where $\alpha$ is the fine-structure constant, $f_{\phi}$ the ALP decay constant, $c_{\gamma}$ the $U(1)_{\mathrm{EM}}$ anomaly coefficient, and $F_{\mu \nu}$ and $\tilde{F}^{\mu \nu} \equiv \epsilon^{\mu \nu \rho \sigma} F_{\rho \sigma} / 2 \sqrt{-g}$ the field strength and its dual. In terms of the electric and magnetic fields we have $F_{\mu \nu} \tilde{F}^{\mu \nu}=-4 \boldsymbol{E} \cdot \boldsymbol{B}$. The natural values of $c_{\gamma}$ is of $\mathcal{O}(1)$, but it can be much larger than unity in a contrived setup $[40,41]$. For simplicity, we assume that the ALP mass is smaller than the current Hubble constant $H_{0} \simeq 10^{-33} \mathrm{eV}$, and neglect the mass in the following, although it can be straightforwardly extended to $m_{\phi} \lesssim 10^{-28} \mathrm{eV}$. As we will see, our scenario not only extends the viable mass region to smaller masses, but also easily satisfies the upper limit on the ALP abundance [42]. The rotation angle of the CMB polarization is related to the change of the ALP field value from the LSS to the present, $\Delta \phi \equiv \phi_{p}-\phi_{\mathrm{LSS}}$, as [26] [Note that there is a sign error after their Eq. (4) of Ref. [26] ].

$$
\beta \simeq 0.42 \operatorname{deg} \times\left(c_{\gamma} \frac{\Delta \phi}{2 \pi f_{\phi}}\right)
$$

Now we introduce an effective Hubble-induced ALP mass from the DM density:

$$
V(\phi)=\frac{1}{2} c_{H} H_{\mathrm{DM}}^{2} \phi^{2},
$$

where we assume $c_{H}$ is a positive constant and $H_{\mathrm{DM}}^{2} \equiv$ $\rho_{\mathrm{DM}} / 3 M_{\mathrm{Pl}}^{2}$, with $\rho_{\mathrm{DM}}$ being the DM energy density, and $M_{\mathrm{Pl}}$ the reduced Planck mass. Here, without loss of generality, the potential minimum $\phi_{\min }$ of the effective potential is set to be at the origin. The Witten effect on the ALP potential in a monopole DM model is actually written in this form as we will see. Alternatively, one may introduce a coupling to the Ricci scalar $R\left(=6\left[(\dot{a} / a)^{2}+\ddot{a} / a\right]\right)$ : $\mathcal{L} \supset-\xi R \phi^{2}$, where $\xi=\mathcal{O}(1)$ is a positive constant. While the effective mass from this operator is negligibly small during the radiation-dominated era because of the conformal symmetry, it is about $\sqrt{6 \xi} H$ during the matterdominated era. This term therefore has a similar effect as Eq. (3) [43]. In the following we use Eq. (3) as an effective potential for the ALP.

We consider a homogeneous ALP in the following, and we will later comment on its quantum fluctuations generated during inflation. The equation of motion for the homogeneous mode is given by

$$
\ddot{\phi}+3 H \dot{\phi}+c_{H} H_{\mathrm{DM}}^{2} \phi=0,
$$

where the dot represents a derivative with respect to time and $H$ is the Hubble parameter obeying $H^{2}=$ $H_{0}^{2}\left(\Omega_{\mathrm{rad}} a^{-4}+\Omega_{\mathrm{mat}} a^{-3}+\Omega_{\Lambda}\right)$ with the present value of the scale factor set to be unity. The effective mass is negligible until the matter-radiation equality because $H_{\mathrm{DM}} \ll H$ during the radiation-dominated era. Then, the ALP starts to move toward the potential minimum after the equality, and it experiences damped oscillations since the effective mass is comparable to the Hubble parameter. As a result, the ALP abundance can be negligibly small at present. Since the recombination occurs soon after the matter-radiation equality, a nonzero amount of isotropic CB is induced.

Numerical results.-We numerically solve the equation of motion (4) with various values of $c_{H}$ and initial conditions. We denote the initial ALP field value as $\phi_{i}$, the potential minimum as $\phi_{\min }(=0)$, and the corresponding angle, $\theta=\phi / f_{\phi}$, with the same indices. Note that $\phi_{i}$ is not necessarily equal to $\phi_{\text {LSS }}$ especially for $c_{H} \gg 1$, since the ALP may start oscillating before recombination. Similarly, $\phi_{p}$ is not necessarily equal to $\phi_{\min }$ especially for $c_{H} \ll 1$, since the mass may be too small for the ALP to settle down at the potential minimum. Since the ALP moves toward the origin until present, we have $\operatorname{sign}[\Delta \phi]=$ $\operatorname{sign}\left[\phi_{\min }-\phi_{i}\right]$ at least for $c_{H} \lesssim 1$. Thus, for a positive $c_{\gamma}$, we should take $\phi_{i}<0$ so as to be consistent with the observational result (2).

In our scenario the ALP tends to start moving slightly before the recombination because the effective mass becomes relevant after the matter-radiation equality. We 


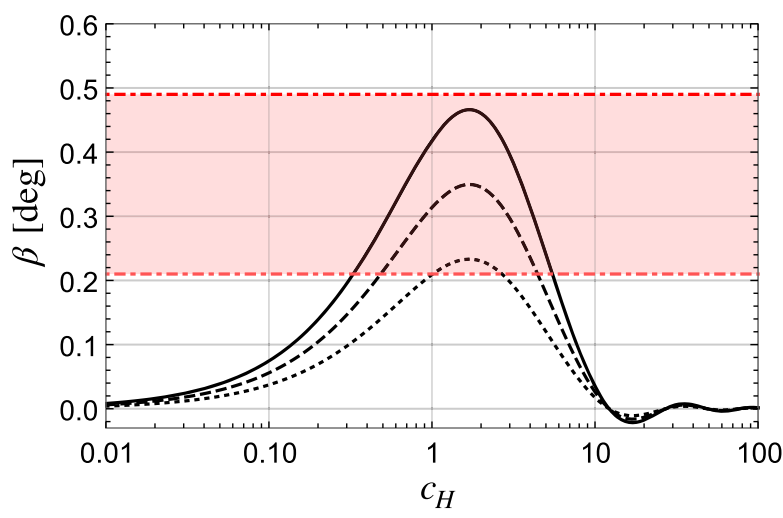

FIG. 1. The predicted rotation angle $\beta$ as a function of $c_{H}$. The solid, dashed, and dotted black lines correspond to $c_{\gamma}=12,9,6$, from top to bottom, respectively. We take the initial condition, $\left(\theta_{i}, \dot{\theta}_{i}\right)=(-1,0)$. The shaded region shows the region favored by the Planck data.

thus need to take account of the thickness of LSS. We estimate the ALP field value at the LSS by weighing it with the visibility function $g(T)$ [44], such as $\left\langle\phi_{\text {LSS }}\right\rangle=$ $\int d T g(T) \phi(T)$. Then, $\beta$ is given by Eq. (2) with $\Delta \phi=\phi_{p}-\left\langle\phi_{\text {LSS }}\right\rangle$.

The resulting rotation angle $\beta$ is shown as a function of $c_{H}$ in Fig. 1. The solid, dashed, and dotted black lines represent the cases with $c_{\gamma}=12,9,6$ from top to bottom, and the red shaded region shows the allowed region by the Planck polarization data. We take the initial condition $\left(\theta_{i}, \dot{\theta}_{i}\right)=(-1,0)$ as an example. One can rescale our result for a different $\theta_{i}$ by using $\beta \propto\left(\theta_{\min }-\theta_{i}\right)$, since the equation of motion Eq. (4) is linear in $\phi$. (This is a good approximation even for a cosine function instead of the quadratic term, as long as the anharmonic effect is small.) As a result, the observational hint for the isotropic $\mathrm{CB}$ can be explained if $c_{\gamma}\left(\theta_{\min }-\theta_{i}\right) \gtrsim 6$ for $c_{H}=\mathcal{O}(1)$.

For $c_{H} \ll 1$, the effective mass is too small and the ALP does not move much even during the matter-dominated era. On the contrary, for $c_{H} \gg 1$, the ALP starts to oscillate well before the matter-radiation equality and it already settles down at the potential minimum by the recombination. Because of the balance between these effects, the rotation angle is maximum at $c_{H} \sim 2$. For a moderately large $c_{H}=\mathcal{O}(10)$, the ALP oscillates a few times around the minimum by the recombination and can have an opposite sign at the LSS. This oscillatory behavior can be seen in Fig. 1.

In Fig. 2 we show a contour of the rotation angle $\beta$ as a function of $c_{H}$ and $c_{\gamma}\left(\theta_{\min }-\theta_{i}\right) /(2 \pi)$. For $c_{H}=\mathcal{O}(1)$, we need $c_{\gamma}\left(\theta_{\min }-\theta_{i}\right) /(2 \pi)=\mathcal{O}(1)$ to obtain the observed value. In other words, no fine-tuning of the initial condition is required in this case, for $c_{\gamma}=\mathcal{O}(1)$. We can see that larger values of $\theta_{\min }-\theta_{i}$ and/or $c_{\gamma}$ are required for larger or smaller values of $c_{H}$.

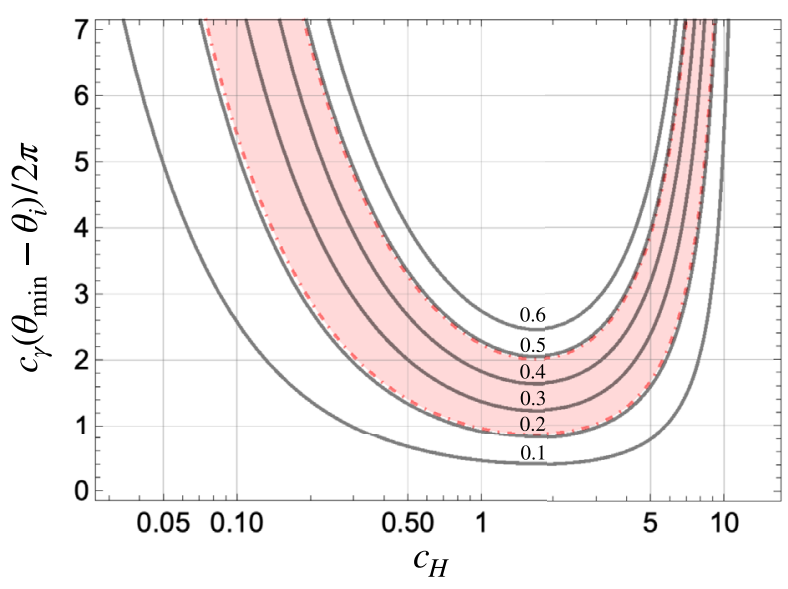

FIG. 2. The contour plot of the rotation angle $\beta$ as a function of $c_{H}$ and $c_{\gamma}\left(\theta_{\min }-\theta_{i}\right) / 2 \pi$. The shaded region shows the observational hint for the isotropic $\mathrm{CB}$.

Effective mass from the Witten effect. - Now we provide an explicit model in which the ALP acquires the effective mass via the Witten effect from hidden monopole DM. A monopole associated with a hidden $U(1)_{H}$ is stable due to its magnetic charge, and is therefore a good candidate for DM. If the ALP has an anomalous coupling to the $U(1)_{H}$ photons, we obtain the aforementioned coupling between the ALP and DM density as in Eq. (3).

First let us see the effect of the $\Theta$ term on monopoles. The Lagrangian of the hidden $U(1)_{H}$ gauge field is given by

$$
\mathcal{L} \supset-\frac{1}{4} X_{\mu \nu} X^{\mu \nu}-\frac{\alpha_{H} \Theta}{8 \pi} X_{\mu \nu} \tilde{X}^{\mu \nu}
$$

where $\alpha_{H}$ is a fine-structure constant for $U(1)_{H}, \Theta$ is a CP violating angle, and $X_{\mu \nu} \equiv \partial_{\mu} X_{\nu}-\partial_{\nu} X_{\mu}$ denotes the field strength of the $U(1)_{H}$ gauge field $X_{\mu}$. Although the second term is a total derivative, it modifies the Maxwell equation as

$$
\nabla \cdot \boldsymbol{E}_{H}=-\frac{\alpha_{H}}{2 \pi} \nabla \cdot\left(\Theta \boldsymbol{B}_{H}\right),
$$

where $\left(\boldsymbol{E}_{H}\right)_{i} \equiv X_{0 i}, \quad\left(\boldsymbol{B}_{H}\right)_{i} \equiv-\epsilon_{i j k} X^{j k} / 2$. A magnetic monopole with a magnetic charge $g_{H}$ sitting at the origin generates a magnetic field, satisfying the Gauss's law, $\nabla \cdot \boldsymbol{B}_{H}=g_{H} \delta^{(3)}(0)$. Equation (6) implies that the magnetic monopole acquires an electric charge proportional to the $\Theta$ parameter, and becomes a dyon. This is known as the Witten effect [45].

Now we introduce the ALP with an anomalous coupling to the $U(1)_{H}$ gauge field. To this end we promote $\Theta$ to the ALP field by the replacement of $\Theta \rightarrow \phi / f_{\phi}$. According to Eq. (6), a nonzero field value of $\phi$ induces an electric field around the monopole. This means that the total electric energy around a monopole $V_{M}$ depends on $\phi$ as 


$$
V_{M} \simeq \frac{\alpha_{H}}{32 \pi^{2} r_{c}} \frac{\phi^{2}}{f_{\phi}^{2}}
$$

where $r_{c}$ is the radius of the monopole core. Considering the 't Hooft-Polyakov monopole [46,47], we have $r_{c} \sim m_{W_{H}}^{-1} \sim\left(\alpha_{H} m_{M}\right)^{-1}$, where $m_{W_{H}}$ and $m_{M}$ are the mass of a heavy gauge field and the monopole, respectively. See Refs. [48-50] for the production and abundances of the hidden monopole and heavy gauge bosons. Taking the spatial average, we obtain the energy density of the ALP ground state as $V(\phi)=n_{M} V_{M}(\phi)$, where $n_{M} \equiv n_{M+}+$ $n_{M-}$ denotes the number density of monopoles and antimonopoles. Thus, the ALP potential is given in the form of Eq. (3) with

$$
c_{H}=3\left(\frac{\rho_{M}}{\rho_{\mathrm{DM}}}\right)\left(\frac{\alpha_{H}}{4 \pi} \frac{M_{\mathrm{Pl}}}{f_{\phi}}\right)^{2},
$$

where $\rho_{M}$ denotes the energy density of the monopole [51].

Here let us comment on an upper bound on $\alpha_{H}$ in our analysis. We expect that monopoles appear from spontaneous symmetry breaking of some non-Abelian gauge theory, such as $\mathrm{SU}(2)_{H}$. Then the instanton effect of $\mathrm{SU}(2)_{H}$ gives rise to an additional mass term about $\phi=0$, though it is suppressed by the instanton exponent, $\sim e^{-\pi / \alpha_{H}}$ [52-54]. This additional mass is negligible [except the effective mass Eq. (3) via the Witten effect] when $\alpha_{H} \lesssim 0.02$. Although our analysis can be straightforwardly extended to a larger gauge coupling if $m_{\phi} \lesssim 10^{-28} \mathrm{eV}$, we assume this inequality for simplicity.

As we have seen above, $c_{H}=\mathcal{O}(1)$ is needed to explain the isotropic CB. This is realized for the ALP decay constant of the order of the grand unified theory scale and $\alpha_{H} \sim \alpha$. To be explicit, for $\alpha_{H}=0.02, \rho_{M}=\rho_{\mathrm{DM}}$, and $f_{\phi}=5 \times 10^{15} \mathrm{GeV}$, we obtain $c_{H} \simeq 2$ from Eq. (8). This shows that the ALP coupled to hidden monopole DM can generate the isotropic $\mathrm{CB}$ without a fine-tuning of $\theta_{i}$, and the grand unified theory scale decay constant suggests that such an ALP may be one of the string axions.

Connection to the QCD axion.-If the QCD axion is coupled to the hidden photons, it acquires the effective mass given by Eq. (3) with the replacement of $c_{H}$ by $c_{H, a}$ (To be precise, it is a combination of the QCD axion and ALP that acquires mass from the Witten effect, but if $f_{a} \ll f_{\phi}$, it is mostly the QCD axion before the QCD phase transition.), which is given by

$$
c_{H, a}=3\left(\frac{\rho_{M}}{\rho_{\mathrm{DM}}}\right)\left(\frac{N_{H}}{N_{\mathrm{DW}}}\right)^{2}\left(\frac{\alpha_{H}}{4 \pi} \frac{M_{\mathrm{Pl}}}{f_{a}}\right)^{2},
$$

where $f_{a}$ is the decay constant for the QCD axion and $N_{\mathrm{DW}}$ and $N_{H}$ are domain wall numbers associated with $S U(3)_{c}$ and $U(1)_{H}$, respectively. The potential minimum of this term is generically different from the one for the QCD vacuum. The effective mass Eq. (3) is negligible at present and does not spoil the success of the Peccei-Quinn (PQ) mechanism [55,56], while it is stronger in the early universe and can affect the evolution of the QCD axion.

In the pre-inflationary PQ symmetry breaking scenario, the effective mass can be used to avoid the isocurvature problem or suppress the QCD axion abundance [57-61]. However, since the hidden gauge coupling considered here is smaller than in the previous studies, the Witten effect might become relevant only for small $f_{a} \lesssim 10^{11} \mathrm{GeV}$.

In the postinflationary PQ symmetry breaking scenario, the effective mass can be used to solve the domain wall problem $[57,60]$. First, we note that cosmic strings form at the time of the PQ symmetry breaking when the temperature is expected to be of order $f_{a}$. Then if the effective mass due to the Witten effect becomes larger than the Hubble parameter before the QCD phase transition, each cosmic string will be attached by a single domain wall if $N_{H}=1$. The cosmic strings therefore soon disappear due to the tension of the domain wall. This sets an approximately homogeneous initial condition on the QCD axion at the QCD phase transition, in which case domain walls do not form when the QCD nonperturbative effect turns on even if $N_{\mathrm{DW}}>1$. Thus we require $c_{H, a} H_{\mathrm{DM}}^{2} \gtrsim H^{2}$ at the QCD phase transition, namely, $c_{H, a} \gtrsim 10^{8}$. This can be satisfied if $f_{a} \ll f_{\phi}$ since $c_{H, a} \sim\left(f_{\phi} / f_{a}\right)^{2} c_{H}$; for $c_{H}=\mathcal{O}(1), f_{\phi}=10^{15-16} \mathrm{GeV}$, and $f_{a}=10^{11} \mathrm{GeV}$, we have $c_{H, a}=\mathcal{O}\left(10^{8-10}\right)$. Therefore, the domain wall problem for the QCD axion can be simultaneously solved by the Witten effect.

Finally, we comment on the deviation of the QCD $\theta$ angle by the Witten effect during the BBN epoch. According to Ref. [62], the success of the BBN theory is spoiled if $\theta_{\mathrm{QCD}}=\mathcal{O}(1)$ during the BBN epoch. In our model, $\theta_{\mathrm{QCD}}$ at the minimum of the potential is less than $10^{-18}$ even at the BBN epoch. The amplitude of the QCD axion is also very small because it starts to oscillate earlier in our model. We thus conclude that the Witten effect on the QCD axion is small enough not to spoil the success of the BBN theory.

Discussion.-So far we have focused on the isotropic $\mathrm{CB}$, but anisotropic $\mathrm{CB}$ is also generated if the ALP acquires quantum fluctuations $\delta \phi \simeq H_{\text {inf }} / 2 \pi$ during inflation. The anisotropic $\mathrm{CB}$ can be sizable especially for a smaller decay constant satisfying $f_{\phi} \sim H_{\text {inf }}\left(\ll 10^{16} \mathrm{GeV}\right)$. Note that the monopole abundance required for our scenario can be correspondingly smaller, and it may only be a fraction of DM. Note also that the ALP with $g_{\phi \gamma \gamma} \gtrsim$ $\mathcal{O}\left(10^{-12}\right) \mathrm{GeV}^{-1}$ can be searched for by Fermi-LAT satellite [63] and solar axion experiments such as IAXO [64-66]).

Let us comment on cosmological aspects of the hidden monopole. The hidden monopole behaves as selfinteracting DM, which could have an interesting effect on 
the DM distribution [67-70]. [The massive $S U(2)_{H}$ gauge bosons also behave as self-interacting DM, although their abundance as well as stability depend on the hidden matter spectrum.] The kinetic mixing between $U(1)_{H}$ and hypercharge is suppressed because it is forbidden by the $S U(2)_{H}$ symmetry at high energy. In the low energy, a tiny kinetic mixing is induced from a higher dimensional operator as a result of the spontaneous breaking of $S U(2)_{H}[57,71,72]$. The hidden monopoles then acquire a small electric charge, though they are well below the current sensitivity [73].

The hidden photon contributes to dark radiation. (In addition, if axions are thermalized, they contribute to dark radiation [74].) Their contributions to the effective neutrino species, $N_{\text {eff }}$, depend on details of the coupling between the hidden sector and the standard model sector, as well as hidden matter spectrum. It predicts $\Delta N_{\text {eff }} \geq 0.054$ and typically $\mathcal{O}(0.1)$. See Refs. $[49,50,60]$ for detailed calculations. The predicted $\Delta N_{\text {eff }}$ may be probed by future observations such as CMB-S4 [75,76] and PICO [77]. Observations of the $21 \mathrm{~cm}$ line radiation from the reionization epoch also have its sensitivity [78].

S. N. acknowledges support from GP-PU at Tohoku University. The present work is supported by JSPS KAKENHI Grants No. 17H02878 (F. T.), No. 20H01894 (F. T.), No. 20H05851 (F. T. and M. Y.), and No. JP20K22344 (M. Y.), World Premier International Research Center Initiative (WPI Initiative), MEXT, Japan. M. Y. was supported by the Leading Initiative for Excellent Young Researchers, MEXT, Japan.

[1] J. Preskill, M. B. Wise, and F. Wilczek, Phys. Lett. 120B, 127 (1983).

[2] L. F. Abbott and P. Sikivie, Phys. Lett. 120B, 133 (1983).

[3] M. Dine and W. Fischler, Phys. Lett. 120B, 137 (1983).

[4] K. Ichikawa, M. Kawasaki, K. Nakayama, M. Senami, and F. Takahashi, J. Cosmol. Astropart. Phys. 05 (2007) 008.

[5] M. Cicoli, J. P. Conlon, and F. Quevedo, Phys. Rev. D 87, 043520 (2013).

[6] T. Higaki and F. Takahashi, J. High Energy Phys. 11 (2012) 125.

[7] T. Higaki, K. Nakayama, and F. Takahashi, J. High Energy Phys. 07 (2013) 005.

[8] M. Cicoli and G. A. Piovano, J. Cosmol. Astropart. Phys. 02 (2019) 048.

[9] F. Takahashi and M. Yamada, J. Cosmol. Astropart. Phys. 07 (2019) 001.

[10] A. Arvanitaki, M. Baryakhtar, and X. Huang, Phys. Rev. D 91, 084011 (2015).

[11] V. Cardoso, O. J. C. Dias, G. S. Hartnett, M. Middleton, P. Pani, and J. E. Santos, J. Cosmol. Astropart. Phys. 03 (2018) 043.

[12] M. J. Stott and D. J. E. Marsh, Phys. Rev. D 98, 083006 (2018).

[13] H. Davoudiasl and P. B. Denton, Phys. Rev. Lett. 123, 021102 (2019).
[14] L. Sun, R. Brito, and M. Isi, Phys. Rev. D 101, 063020 (2020); 102, 089902(E) (2020).

[15] C. Palomba et al., Phys. Rev. Lett. 123, 171101 (2019).

[16] D. Cadamuro and J. Redondo, J. Cosmol. Astropart. Phys. 02 (2012) 032.

[17] P. Arias, D. Cadamuro, M. Goodsell, J. Jaeckel, J. Redondo, and A. Ringwald, J. Cosmol. Astropart. Phys. 06 (2012) 013.

[18] T. Higaki, K. S. Jeong, and F. Takahashi, Phys. Lett. B 733, 25 (2014).

[19] J. Jaeckel, J. Redondo, and A. Ringwald, Phys. Rev. D 89, 103511 (2014).

[20] T. Higaki, N. Kitajima, and F. Takahashi, J. Cosmol. Astropart. Phys. 12 (2014) 004.

[21] M. Kawasaki and T. Yanagida, Phys. Lett. B 399, 45 (1997).

[22] T. Asaka, J. Hashiba, M. Kawasaki, and T. Yanagida, Phys. Rev. D 58, 083509 (1998).

[23] T. Asaka and M. Kawasaki, Phys. Rev. D 60, 123509 (1999).

[24] S. M. Carroll, G. B. Field, and R. Jackiw, Phys. Rev. D 41, 1231 (1990).

[25] S. M. Carroll and G. B. Field, Phys. Rev. D 43, 3789 (1991).

[26] D. Harari and P. Sikivie, Phys. Lett. B 289, 67 (1992).

[27] S. M. Carroll, Phys. Rev. Lett. 81, 3067 (1998).

[28] A. Lue, L.-M. Wang, and M. Kamionkowski, Phys. Rev. Lett. 83, 1506 (1999).

[29] M. Pospelov, A. Ritz, and C. Skordis, Phys. Rev. Lett. 103, 051302 (2009).

[30] M. A. Fedderke, P. W. Graham, and S. Rajendran, Phys. Rev. D 100, 015040 (2019).

[31] P. Agrawal, A. Hook, and J. Huang, J. High Energy Phys. 07 (2020) 138.

[32] Y. Minami and E. Komatsu, Phys. Rev. Lett. 125, 221301 (2020).

[33] Y. Minami, H. Ochi, K. Ichiki, N. Katayama, E. Komatsu, and T. Matsumura, Prog. Theor. Exp. Phys. 2019, $083 E 02$ (2019).

[34] Y. Minami, Prog. Theor. Exp. Phys. 2020, 063E01 (2020).

[35] Y. Minami and E. Komatsu, Prog. Theor. Exp. Phys. 2020, 103E02 (2020).

[36] L. Pogosian, M. Shimon, M. Mewes, and B. Keating, Phys. Rev. D 100, 023507 (2019).

[37] F. Takahashi and W. Yin, J. Cosmol. Astropart. Phys. 04 (2021) 007.

[38] T. Fujita, Y. Minami, K. Murai, and H. Nakatsuka, Phys. Rev. D 103, 063508 (2021).

[39] V. M. Mehta, M. Demirtas, C. Long, D. J. E. Marsh, L. McAllister, and M. J. Stott, J. Cosmol. Astropart. Phys. 07 (2021) 033.

[40] T. Higaki, K. S. Jeong, N. Kitajima, and F. Takahashi, J. High Energy Phys. 06 (2016) 150.

[41] M. Farina, D. Pappadopulo, F. Rompineve, and A. Tesi, J. High Energy Phys. 01 (2017) 095.

[42] R. Hlozek, D. Grin, D. J. E. Marsh, and P. G. Ferreira, Phys. Rev. D 91, 103512 (2015).

[43] F. Takahashi and M. Yamada, J. Cosmol. Astropart. Phys. 10 (2015) 010.

[44] S. Weinberg, Cosmology (Oxford University Press, Oxford, 2008).

[45] E. Witten, Phys. Lett. B 86, 283 (1979). 
[46] G. 't Hooft, Nucl. Phys. B79, 276 (1974).

[47] A. M. Polyakov, JETP Lett. 20, 194 (1974).

[48] H. Murayama and J. Shu, Phys. Lett. B 686, 162 (2010).

[49] S. Baek, P. Ko, and W.-I. Park, J. Cosmol. Astropart. Phys. 10 (2014) 067.

[50] V. V. Khoze and G. Ro, J. High Energy Phys. 10 (2014) 061.

[51] W. Fischler and J. Preskill, Phys. Lett. 125B, 165 (1983).

[52] J. Fuentes-Martín, M. Reig, and A. Vicente, Phys. Rev. D 100, 115028 (2019).

[53] C. Csáki, M. Ruhdorfer, and Y. Shirman, J. High Energy Phys. 04 (2020) 031.

[54] M. A. Buen-Abad and J. Fan, J. High Energy Phys. 12 (2019) 161.

[55] R. D. Peccei and H. R. Quinn, Phys. Rev. Lett. 38, 1440 (1977).

[56] R. D. Peccei and H. R. Quinn, Phys. Rev. D 16, 1791 (1977).

[57] M. Kawasaki, F. Takahashi, and M. Yamada, Phys. Lett. B 753, 677 (2016).

[58] Y. Nomura, S. Rajendran, and F. Sanches, Phys. Rev. Lett. 116, 141803 (2016).

[59] M. Kawasaki, F. Takahashi, and M. Yamada, J. High Energy Phys. 01 (2018) 053.

[60] R. Sato, F. Takahashi, and M. Yamada, Phys. Rev. D 98, 043535 (2018).

[61] S. Nakagawa, F. Takahashi, and M. Yamada, J. Cosmol. Astropart. Phys. 05 (2021) 062.

[62] K. Blum, R. T. D’Agnolo, M. Lisanti, and B. R. Safdi, Phys. Lett. B 737, 30 (2014).
[63] M. Meyer, M. Giannotti, A. Mirizzi, J. Conrad, and M. A. Sánchez-Conde, Phys. Rev. Lett. 118, 011103 (2017).

[64] I. G. Irastorza et al., J. Cosmol. Astropart. Phys. 06 (2011) 013.

[65] E. Armengaud et al., J. Instrum. 9, T05002 (2014).

[66] E. Armengaud et al. (IAXO Collaboration), J. Cosmol. Astropart. Phys. 06 (2019) 047.

[67] M. Rocha, A. H. G. Peter, J. S. Bullock, M. Kaplinghat, S. Garrison-Kimmel, J. Onorbe, and L. A. Moustakas, Mon. Not. R. Astron. Soc. 430, 81 (2013).

[68] M. Vogelsberger, J. Zavala, and A. Loeb, Mon. Not. R. Astron. Soc. 423, 3740 (2012).

[69] A. Kamada, M. Kaplinghat, A. B. Pace, and H.-B. Yu, Phys. Rev. Lett. 119, 111102 (2017).

[70] S. Tulin and H.-B. Yu, Phys. Rep. 730, 1 (2018).

[71] F. Brummer, J. Jaeckel, and V. V. Khoze, J. High Energy Phys. 06 (2009) 037.

[72] F. Brummer and J. Jaeckel, Phys. Lett. B 675, 360 (2009).

[73] E. Del Nobile, M. Nardecchia, and P. Panci, J. Cosmol. Astropart. Phys. 04 (2016) 048.

[74] A. Salvio, A. Strumia, and W. Xue, J. Cosmol. Astropart. Phys. 01 (2014) 011.

[75] K. N. Abazajian et al. (CMB-S4 Collaboration), arXiv: 1610.02743 .

[76] K. Abazajian et al., arXiv:1907.04473.

[77] S. Hanany et al. (NASA PICO Collaboration), arXiv: 1902.10541

[78] Y. Oyama, K. Kohri, and M. Hazumi, J. Cosmol. Astropart. Phys. 02 (2016) 008. 